\title{
DIAGNÓSTICO E CONDUTA CLÍNICA PERANTE DENTES SUPRANUMERÁRIOS LOCALIZADOS EM PRÉ-MAXILA: RELATO DE UM CASO
}

\section{DIAGNOSIS AND CLINICAL APPROACH OF SUPERNUMERARY TEETH IN ANTERIOR MAXILA: A CASE REPORT}

\author{
Marlene Cabral Coimbra da Cruz* \\ Luciana Estevam Simonato* \\ Saygo Tomo* \\ Nagib Pezati Boer* \\ Ana Flávia Sanches Borges"**
}

\section{RESUMO}

Dentre os defeitos do desenvolvimento craniofacial, a Hiperdontia representa uma alteração de número do desenvolvimento dentário relevante, devido a sua alta prevalência e ao seu potencial para comprometer funcional e esteticamente os pacientes acometidos. O presente trabalho descreve o caso de um paciente de 5 anos de idade, acometido pelo desenvolvimento de dois elementos dentários supranumerários em região anterior de maxila. O caso foi diagnosticado a partir de avaliação radiográfica associada à erupção ectópica de um dos elementos supranumerários por face palatina dos incisivos superiores decíduos, os quais se apresentavam com mobilidade fisiológica devido ao desenvolvimento de seus sucessores. O tratamento eleito para o caso foi a extração dos elementos decíduos com mobilidade e do elemento supranumerário erupcionado, optando-se pela remoção do elemento supranumerário incluso em momento posterior, uma vez que este não se encontrava associado a nenhum comprometimento funcional ou estético e também pelo fato de que a remoção do mesmo geraria alta morbidade ao paciente pediátrico. O caso descrito salienta a importância do cirurgião no acompanhamento do desenvolvimento craniofacial humano, uma vez que este pode ser acompanhado de distúrbios significativos. Além disso, o presente caso alerta a comunidade odontológica para a importância do bom planejamento e conduta terapêutica adequados para cada caso.

Descritores: Dente Supranumerário • Diagnóstico Bucal • Cirurgia Bucal.

\section{A B S TRACT}

Among the defects of craniofacial development, Hyperdontia represents a relevant number of changes of the tooth development, due to its high prevalence and its potential for functional and aesthetically compromising the affected patients. This paper describes the case of a 5 years-old patient, affected by the development of two dental elements supernumerary in the anterior maxilla. The case was diagnosed from radiographic evaluation associated with clinical aspect of ectopic eruption of the supernumerary elements by palatal face of the upper deciduous incisors, which presented with physiological mobility due to the development of their successors. The treatment chosen for the case was the extraction of the deciduous elements mobility and supernumerary element erupted opting up by removal of the supernumerary element included at a later stage, since this was not associate with any functional or esthetic damage, and also by the fact that the removal of that would generate high morbidity to pediatric patient. This case emphasizes the importance of dental surgeon in monitoring of human craniofacial development, since this may be accompanied by significant disorders. Moreover, this case alerts the dental community to the importance of good planning and adequate therapeutic approach for each case.

Descriptors: Tooth, Supernumerary • Diagnosis, Oral • Surgery, Oral.

* Professora do curso de Odontologia, Universidade Camilo Castelo Branco - UNICASTELO, Fernandópolis, SP.

** Aluno de Graduação, curso de Odontologia, Universidade Camilo Castelo Branco - UNICASTELO, Fernandópolis, SP.

*** Professora do departamento de Dentística, Endodontia e Materiais Odontológicos, Faculdade de Odontologia de Bauru - FOB/USP. 


\section{N T R O D U Ç Ã O}

As anormalidades dentárias são responsáveis por uma significante parcela dentre os mais variados defeitos do desenvolvimento craniofacial humano, podendo estar associadas ao número ou morfologia dos elementos dentários que compõem a dentição humana ${ }^{1}$. As anormalidades no número de elementos dentários representam um desafio ao Cirurgião-Dentista, uma vez que podem ser responsáveis por diversas complicações funcionais e estéticas e podem ser classificadas em hiperdontia, quando ocorre um aumento no número de dentes, e hipodontia quando surge uma redução no número de elementos dentários em um indivíduo ${ }^{2,3}$.

A hiperdontia é, portanto, caracterizada pela presença de dentes supranumerários, que podem ser definidos como elementos de estrutura dentária que excedem a fórmula dental fisiológica. Os elementos supranumerários podem ser únicos ou múltiplos em um mesmo indivíduo ${ }^{1}$ ${ }^{4}$. Essa condição pode ainda ocorrer tanto na dentição decídua como na dentição permanente, entretanto é mais comum na dentição permanente, com índices de prevalência variando de $1,5 \%$ a $3,5 \%$, ao passo que, na dentição decídua, tais índices variam de $0,3 \%$ a $0.8 \%{ }^{5,6}$.

Em contraste com a hipodontia, a presença de elementos supranumerários demonstra certa predileção pelo gênero masculino, com uma proporção homem:mulher de aproximadamente $2: 1^{7}$.

A etiologia responsável pela ocorrência de elementos dentários supranumerários é ainda pouco conhecida, sendo atualmente considerada multifatorial, na qual fatores genéticos e também relacionados ao desenvolvimento parecem estar associados $^{1}$. Apesar de ter sua etiologia mal conhecida, os elementos dentários supranumerários podem ser classificados considerando-se alguns parâmetros associados à sua ocorrência e, portanto, são classificados de acordo com sua localização, morfologia, orientação e posição ${ }^{7}$ Tal classificação é de fundamental importância para o diagnóstico e plano terapêutico para os elementos supranumerários, além de facilitar a comunicação entre os profis- sionais odontólogos, visto que muitas vezes o tratamento para esses casos envolve uma abordagem multidisciplinar ${ }^{3,7}$.

A abordagem terapêutica para os elementos dentários supranumerários consiste basicamente na remoção cirúrgica desses $^{3}$. Entretanto, diversos fatores devem ser avaliados, como a idade do paciente, as complicações causadas pela presença destes supranumerários e a possibilidade de posicionamento funcional desses elementos no arco dental ${ }^{8,9}$.

\section{RELATO DE CASO}

Paciente M.V.C., gênero masculino, 5 anos e 7 meses de idade, leucoderma, compareceu a atendimento odontológico para extração do elemento dentário 61 devido a erupção de seu sucessor permanente por face palatina. Ao exame clínico intraoral notou-se mobilidade de grau I nos elementos 61 e 71 e a presença de um elemento dentário com aspecto morfológico de canino permanente com coroa irrompida pela face palatina do elemen-

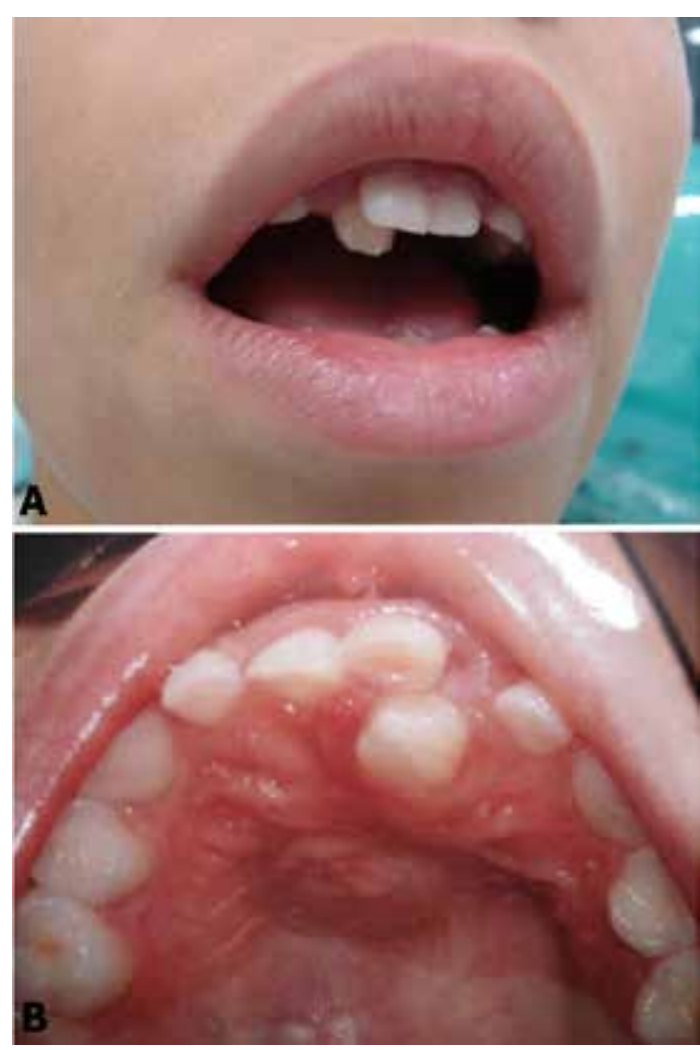

Imagem 1. Aspecto clínico intrabucal inicial. Note-se elemento supranumerário com aspecto de canino erupcionado pela face palatina do elemento 61. (A) Vista frontal. (B) Vista inferior.
CRUZ MCC

Simonato LE

TOMOS

BOER NP

BORGES AFS

DIAGNÓSTICO E CONDUTA CLÍNICA PERANTE DENTES SUPRANUMERÁRIOS LOCALIZADOS EM PRÉ-MAXILA :

RELATO DE UM CASO

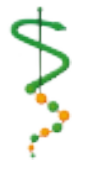

REV, ODONTOL,

UNIV. CID, SÃO PAULO

$2015 ; 27(3): 258-$ 62, SET-DEZ 

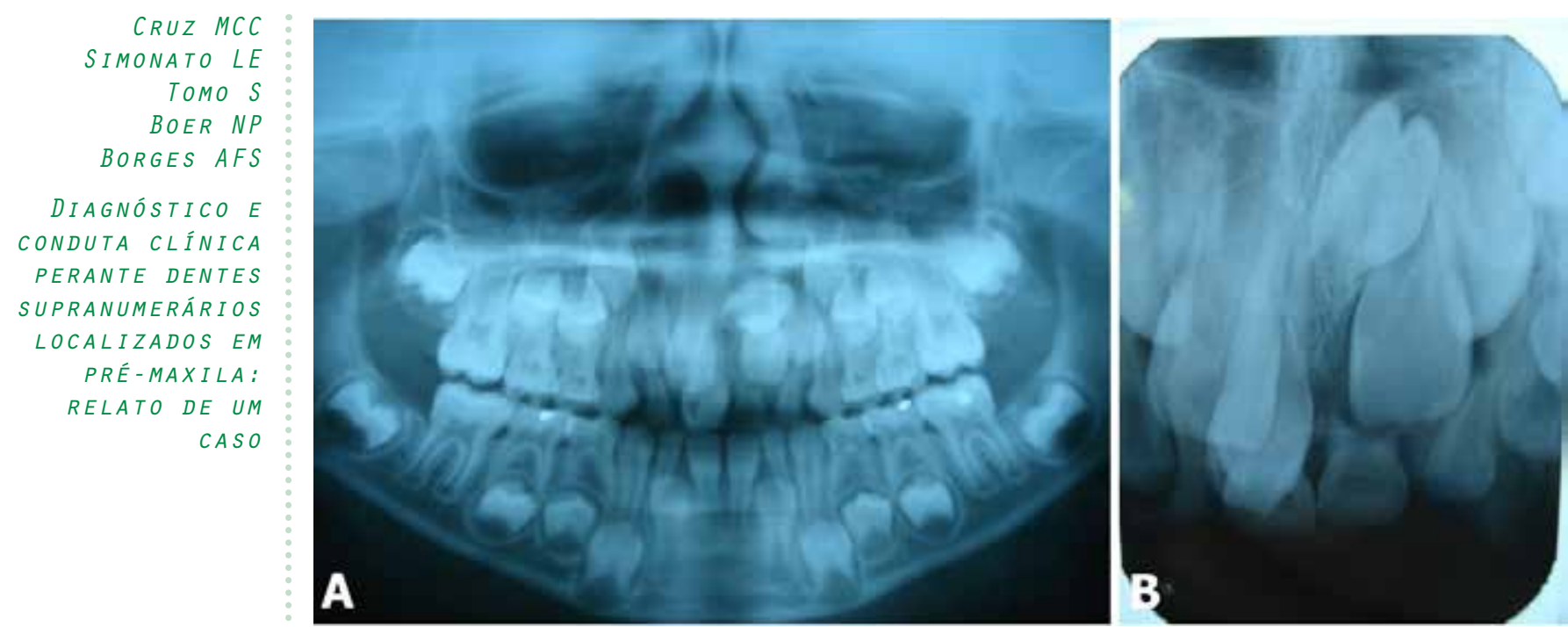

Imagem 2. Aspecto radiográfico inicial. (A) Tomada radiográfica panorâmica. (B) Tomada radiográfica periapical.

to 61 (Imagem 1). Além disso, foi notada a presença de uma tumefação localizada posteriormente ao elemento semelhante a canino irrompido (Imagem 1).

A avaliação radiográfica inicial realizada por radiografia periapical da região de incisivos superiores revelou a presença de dois elementos dentários supranumerários, sendo que o elemento irrompido encontrava-se em posição normal, ao passo que o elemento impactado encontrava-se em posição invertida. A avaliação radiográfica panorâmica, realizada posteriormente ao exame radiográfico periapical, não revelou a presença de nenhum outro elemento dentário supranumerário além dos elementos localizados na pré-maxila (Imagem 2).

O tratamento de escolha para o caso foi a remoção cirúrgica dos incisivos centrais superiores decíduos e do elemento

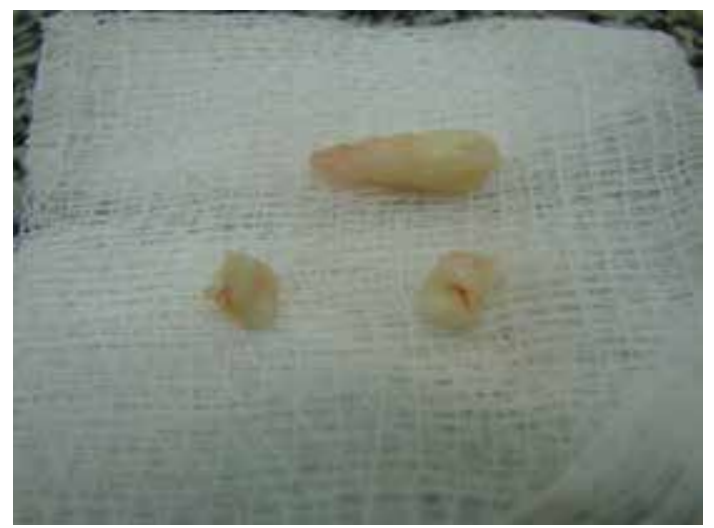

UNIV. CID, SÃO PAULO

$2015 ; 27(3): 258-$ 62, SET-DEZ supranumerário já erupcionado, optando-se pela remoção cirúrgica do elemento supranumerário impactado em momento posterior (Imagem 3).

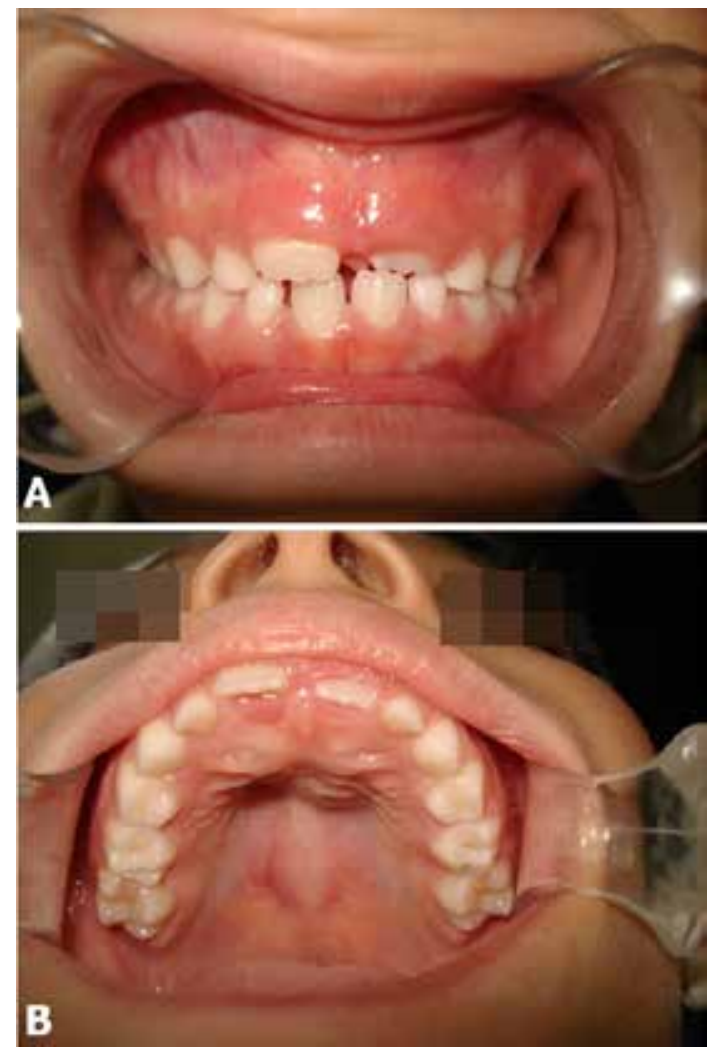

Imagem 4. Aspecto clínico 3 meses após o procedimento cirúrgico. Note-se erupção fisiológica dos incisivos superiores permanentes. (A) Vista frontal. (B) Vista inferior. Note-se discreta tumefação em região de linha média do palato duro causada pela presença do elemento supranumerário incluso. 
Após três meses do procedimento cirúrgico inicial, notou-se a erupção típica dos incisivos superiores permanentes, ao passo que a presença do supranumerário impactado não gerou nenhuma complicação até o momento (Imagem 4).

\section{I SCUSSÃO}

As anormalidades relacionadas ao número de elementos dentários têm se demonstrado cada vez mais prevalentes na população em geral, o que pode ser atribuído a fatores genéticos e ambientais ${ }^{1-3}$. Clinicamente, tais anomalias representam desafios ao Cirurgião-Dentista, necessitando, muitas vezes, de abordagem terapêutica complexa multidisciplinar ${ }^{8,9}$.

Os dentes supranumerários que caracterizam a hiperdontia podem estar presentes como elementos únicos, no entanto a presença de mais de um elemento dentário supranumerário pode também ser observada ${ }^{7}$. Em estudo realizado por Mossas et al. ${ }^{7}$ (2014), os autores relataram que em uma amostra de 101 pacientes, apenas $15,8 \%$ foram acometidos por dois elementos supranumerários, como no caso descrito no presente trabalho, ao passo que $80,5 \%$ foram acometidos por apenas um elemento supranumerário. Além disso, a literatura é persistente em afirmar a predileção da hiperdontia em acometer pacientes do gênero masculino, com relação homem:mulher que varia de 1,5:1 a $3: 1^{4,5}$ e, dessa forma, o caso descrito neste trabalho corrobora com esses dados.

A presença de elementos dentários supranumerários pode ser observada tanto em casos nos quais esses elementos se encontram erupcionados, bem posicionados no arco dental ou em posição atípica, como em casos nos quais tais elementos se encontram impactados ${ }^{5,7}$. No caso descrito neste trabalho, o paciente apresentou-se com um dos elementos supranumerários erupcionado em sua pré-maxila, ao passo que outro elemento supranumerário encontrava-se impactado em posição horizontal posteriormente ao elemento erupcionado. Kashyap et al. ${ }^{4}$ (2015)relataram que, na maioria dos casos $(92,19 \%)$, os elementos supranumerários são encontrados impactados, o que se deve ao fato de que muitas vezes não há espaço para a erupção dos mesmos, ao passo que apenas $7,81 \%$ dos elementos supranumerários encontram-se irrompidos na cavidade oral, na maioria das vezes fora do arco dental ou causando desalinhamento dos elementos fisiológicos dos pacientes acometidos.

No mesmo estudo, os autores relataram que a região posterior da maxila foi a região anatômica mais comumente afetada pela presença de elementos dentários supranumerários, seguida pela região posterior da mandíbula ${ }^{4}$. Em contraste, Anegundi et al. ${ }^{5}$ (2014)relataram maior prevalência $(92,53 \%)$ de elementos dentários supranumerários na região anterior da maxila, sendo esta a localização acometida pelos elementos supranumerários no caso descrito neste trabalho. Além disso, no mesmo estudo, os autores relataram que a maioria dos pacientes afetados pela hiperdontia encontrava-se em estágio de dentição mista, assim como o paciente descrito neste trabalho ${ }^{5}$.

A abordagem terapêutica para os casos de hiperdontia basicamente consiste na remoção cirúrgica dos elementos supranumerários presentes, e, quando necessário, reposicionamento ortodôntico dos elementos fisiológicos do paciente ${ }^{8,9}$. Entretanto, tal remoção cirúrgica pode não ser indicada ou necessária em alguns $\operatorname{casos}^{8,9}$. Em revisão realizada por Parolia et al. ${ }^{3}$ (2011)os autores descreveram que certos fatores devem ser cuidadosamente avaliados antes que conduta cirúrgica seja adotada. Dentre estes, os principais fatores que levam à indicação de remoção cirúrgica dos elementos supranumerários são: o elemento supranumerário causar ou estar associado a algum processo patológico e a presença de um elemento supranumerário estar prejudicando a erupção ou posicionamento dos elementos fisiológicos do paciente 3 . Dessa forma, no presente caso, a remoção cirúrgica foi eleita apenas para o elemento supranumerário erupcionado na pré-maxila, uma vez que este causava comprometimento estético e funcional ao paciente, além de ser um fator de risco para irritação traumática crônica na língua do mesmo. O elemento impactado posteriormente ao elemento erupcionado foi mantido, uma
CRUZ MCC

SIMONATO LE

TOMOS

BOER NP

BORGES AFS

DIAGNÓSTICO E

CONDUTA CLÍNICA

PERANTE DENTES

SUPRANUMERÁRIOS

LocalizadOS EM

PRÉ-MAXILA:

RELATO DE UM

CASO

261

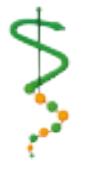

REV, ODONTOL.

UNIV, CID, SÃO

PAULO

$2015 ; 27(3): 258-$

62, SET-DEZ 
CRUZ MCC

SIMONATO LE

TOMO S

BOER NP

BORGES AFS

DIAGNÓSTICO E CONDUTA CLÍNICA PERANTE DENTES SUPRANUMERÁRIOS LOCALIZADOS EM $P R E$-MAXILA : RELATO DE UM CASO

\section{ISSN 1983-5183}

vez que não causava nenhum comprometimento estético ou funcional, e a remoção cirúrgica para esse elemento causaria grande morbidade trans e pós-cirúrgica ao paciente.

\section{CONCLUSÃO}

O caso relatado alerta a comunidade odontológica para a relevância clínica da hiperdontia, que apresenta potencial para exercer influência negativa sobre os ele- mentos dentários fisiológicos dos pacientes acometidos por essa condição. Além disso, este caso também salienta a importância de avaliação criteriosa para eleição de conduta clínica adequada para cada caso.

\section{CONFLITOS DE INTERESSE}

Os autores declaram não haver nenhum conflito de interesse referente ao presente trabalho.

\section{REFERÊNCIAS}

1. Mallineni SK, editor. Supernumerary teeth: Review of the literature with recent updates. Conference Papers in Science; 2014: Hindawi Publishing Corporation.

2. Neville BW, Damm DD, Allen CM, Bouquot JE. Patologia oral e maxilofacial. 3 ed. Rio de Janeiro: Elsevier; 2009.

3. Parolia A, Kundabala M, Dahal M, Mohan M, Thomas MS. Management of supernumerary teeth. I Conserv Dent 2011 Jul;14(3):221-4.

4. Kashyap RR, Kashyap RS, Kini R, Naik $V$. Prevalence of hyperdontia in nonsyndromic South Indian population: An institutional analysis. Indian J Dent 2015 Jul-Sep;6(3):135-8.

5. Anegundi RT, Tegginmani VS, Battepati P, Tavargeri A, Patil S, Trasad V, et al. Prevalence and characteristics of supernumerary teeth in a non-syndromic South Indian pediatric population. J Indian Soc Pedod Prev Dent 2014 Jan-Mar;32(1):9-12.
6. Gopakumar D, Thomas J, Ranimol P, Vineet D, Thomas S, Nair V. Prevalence of supernumerary teeth in permanente dentition among patients attending a dental college in South Kerala: a pilot study. J Indian Acad Oral Med Rad 2014 26(11):42-5.

7. Mossaz J, Kloukos D, Pandis N, Suter VG, Katsaros C, Bornstein MM. Morphologic characteristics, location, and associated complications of maxillary and mandibular supernumerary teeth as evaluated using cone beam computed tomography. The European Journal of Orthodontics 2014 36(6):708-18.

8. Ata-Ali F, Ata-Ali J, Peñarrocha-Oltra D, Peñarrocha-Diago M. Prevalence, etiology, diagnosis, treatment and complications of supernumerary teeth. Journal of clinical and experimental dentistry 2014 6(4):e414.

9. Jain S, Jain P. Surgical Management of Palatally Placed Impacted Mesiodens: A Case Report. IJSS 2015 1(11):70.

Recebido em 29/02/2016

Aceito em 08/03/2016
REV, ODONTOL, UNIV. CID. SÃO PAULO: $2015 ; 27(3): 258-$ 62 , SET-DEZ 